\title{
Impacto psicológico de la pandemia por COVID-19 en los psicoterapeutas españoles. Nuevos desafíos en psicoterapia psicoanalítica
}

\author{
Ricardo Gallego Luis ${ }^{1}$ y Rosario Castaño Catalá ${ }^{2}$ \\ FEAP
}

\begin{abstract}
Todos los profesionales de la salud estamos siendo testigos en primera línea de esta crisis de salud sin precedentes a nivel mundial, estamos inmersos en lo que podemos llamar un trauma social y colectivo de grandes proporciones, y cuando se está inmerso en un trauma no se tiene la suficiente distancia objetiva ni los datos fidedignos para hacer un análisis riguroso. Por ello, en la primavera pasada, mientras salíamos poco a poco del confinamiento en todo el territorio nacional, llevamos a cabo el ESTUDIO FEAP COVID-19, un análisis del impacto psicológico de la pandemia en psicoterapeutas españoles. Han participado en este estudio online todas las Secciones de la Federación Española de Asociaciones de Psicoterapeutas, FEAP con sus diferentes asociaciones, y hemos recogido lo que piensan 554 personas de nuestro colectivo, lo hemos analizado y aquí compartimos algunos datos interesantes. ¿Cómo nos está afectando, y qué desafíos supone en la práctica clínica el hecho de ser testigos de un sufrimiento que también nos afecta a nosotros como parte que somos de nuestra sociedad?
\end{abstract}

Palabras clave: Covid-19, Psicoterapeutas, Psicoterapia Psicoanalítica

All health professionals are witnessing this unprecedented health crisis worldwide, we are immersed in what we can call a social and collective trauma of great proportions, and when you are immersed in a trauma you do not have sufficient objective distance and reliable data to do a rigorous analysis. For this reason, last spring, as we gradually emerged from confinement throughout the national territory, we carried out the FEAP COVID-19 STUDY, an analysis of the psychological impact of the pandemic on Spanish psychotherapists. All Sections of the Spanish Federation of Associations of Psychotherapists, FEAP with their different associations have participated in this online study, and we have collected what 554 people from our group think, we have analyzed it and here we share some interesting data. How is it affecting us, and what challenges does it pose in clinical practice to witness suffering that also affects us as part of our society?

Key Words: Covid-19, Psychotherapists, Psychoanalytic Psychotherapy

English Title: Psychological impact of the COVID-19 pandemic in Spanish psychotherapists. New challenges in psychoanalytic psychotherapy

\section{Cita bibliográfica / Reference citation:}

Gallego Luis, R. y Castaño Catalá, R. (2021). Impacto psicológico de la pandemia por COVID-19 en los psicoterapeutas españoles. Nuevos desafíos en psicoterapia psicoanalítica. Clínica e Investigación Relacional, 15 (1): 206-215. [ISSN 1988-2939] [Recuperado de www.ceir.info ] DOI: 10.21110/19882939.2021.150110

\footnotetext{
${ }^{1}$ Ricardo Gallego Luis, médico de familia, sexólogo y psicoterapeuta psicoanalítico. Miembro de la junta directiva del CEAP, Centro de Estudio y Aplicación del Psicoanálisis de Madrid. Coordinador del estudio FEAP COVID-19.

${ }^{2}$ Rosario Castaño Catalá, psicóloga especialista en psicología clínica, sexóloga, y psicoterapeuta relacional, es miembro de la junta directiva del IPR, Instituto Psicoterapia Relacional, con labores docentes y de supervisión, presidenta de la Sección de Psicoterapias Psicoanalíticas de la FEAP.
} 
Ha pasado ya casi un año desde que se declaró el Estado de Alarma en España, y seguimos inmersos en lo que se ha denominado la tercera ola del coronavirus ${ }^{1}$. Esta pandemia supone un reto mundial a nivel sanitario pues afecta en principio a la salud física, pero también al bienestar emocional y a la salud mental, y ha supuesto grandes cambios en la vida cotidiana para la mayoría de personas y familias: teletrabajo, incertidumbre laboral, desempleo, pérdidas económicas ... la educación, el contacto familiar, el entretenimiento o la formación online, así como un incremento del comercio electrónico ${ }^{2}$, entre otros cambios.

Hoy en día, la sociedad occidental está haciendo frente a este desafío sanitario gracias al empeño de los profesionales de la salud, y al miedo y la necesidad de estar unidos con un único objetivo: prevenir esta enfermedad y eliminar lo antes posible los contagios masivos, sin embargo, este sentimiento de "estar todos navegando en la misma dirección" se va diluyendo a medida que pasa el tiempo y las desigualdades y contratiempos nos van afectando a cada uno de diferente manera, produciéndose un mayor agotamiento psicológico entre la población general con síntomas de tristeza, soledad, ansiedad y miedo, lo que la Organización Mundial de la Salud, OMS ha denominado la Fatiga Pandémica ${ }^{3}$.

Todos los profesionales de la salud estamos siendo testigos en primera línea de esta crisis de salud sin precedentes a nivel mundial, estamos inmersos en lo que podemos llamar un trauma social y colectivo de grandes proporciones, y cuando se está inmerso en un trauma no se tiene la suficiente distancia objetiva ni los datos fidedignos para hacer una análisis riguroso. Por ello, en la primavera pasada, mientras salíamos poco a poco del confinamiento 4 en todo el territorio nacional, llevamos a cabo el ESTUDIO FEAP COVID-19, un análisis del impacto psicológico de la pandemia en psicoterapeutas españoles 5 . Han participado en este estudio online todas las Secciones de la Federación Española de Asociaciones de Psicoterapeutas, FEAP con sus diferentes asociaciones, y hemos recogido lo que piensan 554 personas de nuestro colectivo, lo hemos analizado y aquí compartimos algunos datos interesantes.

¿Cómo nos está afectando, y qué desafíos supone en la práctica clínica el hecho de ser testigos de un sufrimiento que también nos afecta a nosotros como parte que somos de nuestra sociedad?

Ante un trauma personal y colectivo nos enfrentamos a las emociones (ver Rodríguez Sutil, $2014)^{6}$ una emoción siempre es intencional, es la que colorea nuestra razón y nuestras decisiones, evalúa los pros y los contras, e incluye no solo la razón, también las creencias, los prejuicios, la experiencia, el carácter y la personalidad de cada uno, por lo tanto es consciente y también, en gran parte inconsciente. 
En el sector salud el nuevo humanismo ya se estaba imponiendo con muchas dificultades, ${ }^{7}$ esa perspectiva que descarta por completo la falacia cientificista, según la cual todo ha de ser verificado, comprobado, y examinado de acuerdo con la metodología de las ciencias exactas, y que hemos de considerar al ser humano como un ente solo de razón, en clave reduccionista y positivista, olvidando que el ser humano es contingente, mítico y contradictorio, no es ni ángel ni bestia. (ver Peteiro, 2010; Gadamer, 2017)

Cuando se produce un suceso inesperado y traumático, las emociones se vuelven más intensas, el funcionamiento cognitivo se ve anulado, apareciendo la memoria afectiva y episódica con sensaciones somáticas, imágenes visuales que vuelven con síntomas físicos y flashbacks sin significado narrativo, de ahí las pesadillas y los sueños raros que manifiestan la mayoría de las personas, incluidos los pacientes; hay una invocación del destino, del mito, de los dioses; nuestra primera reacción es volvernos hacia/refugiarnos en un pensamiento mágico más propio del pensamiento infantil. Se entienden estos hashtags \#destino, \#astrología \#universo que se buscaron ampliamente durante el confinamiento en las redes como una forma de bucear en sensaciones y sentimientos que no responden a nuestra lógica habitual pero se buscan casi de forma compulsiva, como un modo de entender y lograr la seguridad perdida.

En un primer momento ante la pandemia hubo una reacción normal de sorpresa y miedo, era un confinamiento tranquilo y resignado. Luego vino el desconfinamiento, y otros reconfinamientos ${ }^{8}$... esta situación se ha ido prolongando y es muy frecuente ver reacciones, o bien de hiperactividad para no sentir dolor y angustia, o bien de negación, y disociación de la realidad. Hay mucho enfado, hartazgo, angustia, miedo, desilusión, frustración, apatía, falta de una línea de horizonte clara. La realidad que vivimos es dura. Hay mucho desencanto con el gobierno y las instituciones.

Ante un trauma $^{9}$ (ver Coderch, 2012 pp 302) suele haber una ruptura del ritmo del espacio y tiempo, que son categorías básicas de la experiencia humana de las que no discutimos sus significados, los damos por sentado, nos organizamos en torno a un tiempo rutinario de horas, días, años ... y en torno también a un tiempo y un espacio social con la familia, amigos, trabajo ... estos ritmos son como el aire que respiramos, del que no somos conscientes hasta que empezamos a notar la falta de oxígeno.

Esa ruptura se refleja en la práctica clínica, nos hemos visto obligados a una revisión detallada de los procedimientos y protocolos internos de calidad, empezando por una serie de cambios en la distribución de los espacios para que sean más amplios y seguros y a utilizar internet como alternativa a la consulta y a las sesiones presenciales. Según el estudio de FEAP, hoy día la mayoría de nosotros utilizamos la psicoterapia a distancia (93\%). Antes del 
confinamiento tan solo la usábamos un $47 \%$. Hemos conseguido adaptarnos con rapidez y eficacia al cambio que se nos propuso de la noche a la mañana. Es indudable que pasar a la modalidad online ha tenido un coste en términos de adaptación al nuevo encuadre. Hemos tenido que aprender, nos hemos cansado más, y también ha tenido un impacto en nuestra economía. En el confinamiento, en más del $85 \%$ de nuestras consultas ha habido pacientes que no han aceptado este cambio de modalidad $^{10}$.

El uso obligatorio y necesario de la mascarilla, la distancia social, la falta de contacto en saludos y despedidas, y el uso de la tecnología están provocando transformaciones que vamos analizando en las diferentes reuniones y simposios online, algo que ya está siendo motivo de debates en los entornos de la psicoterapia desde que se implantase la consulta telemática, preguntas como: la mayor o menor exposición de la privacidad de paciente y terapeuta, la mayor o menor intimidad, o accesibilidad, el manejo de la inmediatez, los horarios, la aparente mayor flexibilidad, así como la relación con el espacio, con la corporalidad, el uso de la imagen, ... en definitiva la instauración de nuevos protocolos para nuevas situaciones.

Los psicoterapeutas somos a la vez testigos y afectados por los discursos políticos y movimientos sociales que ponen de manifiesto una sociedad cada vez más dividida y polarizada en la que el miedo al Otro, al diferente, parece dominarlo todo (Bauman, 2010) y en la que se ha instaurado la mundialización y la ideología de la salud en una atmosfera de ansiedad y decepción (Lipovestsky, 2008).

Desde la aparición de la pandemia se están produciendo situaciones que ya existían y que se han exacerbado considerablemente: la cada vez más importante brecha de género, desigualdades, autoritarismo, homofobia, racismo, misoginia, nacionalismos, explotación de seres humanos, o la tan mencionada crisis climática. No obstante, esta crisis sanitaria también nos ha traído la necesidad de adaptarnos a una vida que requiere de cualidades que asociamos más con lo femenino, con el contacto con la naturaleza, con los cuidados y la diversidad, con el hecho de sentirnos vulnerables y dependientes, y sabernos relacionales, y con aquello que resalta lo colectivo y la pertenencia de grupo, a pesar de que la imagen de un mundo globalizado e interconectado no ha traído consigo una nueva idea del "nosotros", dependemos unos de otros más que nunca y sin embargo no sabemos decir "nosotros"...

... entre el yo y el todo no sabemos dónde situar nuestros vínculos, complicidades, alianzas y solidaridades (Garcés, 2013, pp 21).

Vemos este mundo hiperconectado como un mundo fragmentado en el que se fomenta la exclusión, y como explica Bauman, 2010, se crea una nueva clase media que padece una aguda incertidumbre existencial, ansiedad y miedo, en medio de una sociedad claramente 
dividida que lucha entre dos extremos: la criminalización de aquellos que se hallan por debajo de la norma idealizada y el respeto por los que se someten al orden establecido y a la ley.

Los psicoterapeutas, como profesionales de la salud, nos sentimos relativamente protegidos y privilegiados, pero también vulnerables; y este reconocimiento de nuestra propia vulnerabilidad nos puede ayudar a entender mejor esos conflictos sociales y políticos, así como el sufrimiento individual que cada uno filtra a través de su propia historia e idiosincrasia; ser testigos nos coloca en un lugar de privilegio pero también de sostén y de responsabilidad en una circunstancias que nos obligan a soportar nuestro dolor y el ajeno, lo que precisa una mayor atención a la realidad actual del paciente. Sabemos que la salud mental depende en gran medida de las expectativas vitales de cada uno, pero también sabemos que los recursos materiales y humanos de los que se dispone son fundamentales.

En estas circunstancias, en el trabajo clínico cobra más importancia que nunca el reconocimiento de la propia subjetividad y de la escucha intersubjetiva, como dice Mitchell, 1993, pp 13, desde el modelo relacional...

... no somos ingenuos, la dimensión intrasubjetiva también es importante, ... no obstante las relaciones con los demás y no las pulsiones son la materia prima de la vida mental;

y como defienden Orange, Atwood y Stolorow (2012) la escucha intersubjetiva nos permite analizar los conflictos desde el contexto emocional en el cual se dan y se mantienen configuraciones subjetivas particulares, y no enfocar el origen y la perpetuación de la psicopatología y el sufrimiento únicamente dentro del paciente. ${ }^{11}$

Todos somos víctimas potenciales de la pandemia, bien porque nos podemos infectar, bien porque sufriremos el miedo, la incertidumbre y las restricciones que nos impone. Hemos recogido las variables del estudio que se asocian a una mejoría en la puntuación del $\mathrm{GHQ}_{12}{ }^{12}$ , por ejemplo, a mayor edad, menor impacto en la salud mental; parece que la experiencia ayuda a saber gestionar mejor los síntomas ansiosos y depresivos, o considerar que "mi estado de salud es bueno o muy bueno", impacta positivamente en mi estado de salud mental (92\%).

También mejora el score del GHQ-12 tener una visión optimista, como considerar "nada o poco probable" el contagio por COVID-19 (55\% participantes), creer que el impacto económico sobre mi vida no será significativo ( $87 \%$ participantes), tener una visión optimista del futuro, no utilizar psicofármacos $(88,5 \%$ participantes) o no tener un consumo aumentado de comida (71,5\% participantes). 
En general, vemos que la actitud tiene una importancia fundamental en el impacto sobre la salud mental. Por ejemplo, aquellas personas que se han contagiado de la COVID-19 (7\% en nuestro estudio) ${ }^{13}$ no presentan un impacto negativo en su salud mental, sin embargo, aquellas que no se han contagiado pero creen que se pueden contagiar, es decir, que tienen miedo, sí muestran una puntuación más alta de su GHO12 (impacto negativo en su salud mental). De igual manera ocurre entre aquellos que viven solos (sin impacto en su salud mental) y aquellos que se sienten solos (con impacto), o entre aquellos que ya han sufrido un importante recorte en su economía (sin impacto) y entre aquellos que temen que ese impacto se produzca en el futuro (con impacto).

Estos datos nos hacen pensar en las fantasías pero no en las fantasías basadas exclusivamente en deseos insatisfechos ni como estados reprimidos del soñar despierto tal como las describe Freud en 1911 (pp. 1638) sino como el funcionamiento de esquemas inconscientes preformados y formados en la matriz intersubjetiva niño-cuidador y a partir de los cuales se asimilan las experiencias actuales. (Mitchell, 1993; Mitchell y Black, 2004; Storolow, y Atwood, 2004; Coderch, 2014). Existe una cierta semejanza del self con tipos de fantasía sobre uno mismo, en especial inconscientes, tal como lo expone Rodríguez Sutil, 2010; Castaño, 2011, y abordar estas fantasías en el proceso terapéutico constituye una gran ayuda para entender expectativas, temores y ansiedades ante una situación tan especial como la de la COVIDig.

Aparte de la actitud individual ante el mundo y las fantasías sobre uno mismo y los otros, se están abordando en la clínica la culpa y el duelo ${ }^{14}$ por la muerte de algún ser querido o cercano, y también lo que podríamos denominar "el duelo colectivo". Pensemos en esos ataúdes anónimos ${ }^{15}$ que, día a día y durante todo este año, son mostrados a través de los medios de comunicación, y que se asocian con personas que se han enfrentado a la muerte en la más absoluta soledad; unas imágenes que entran en el espacio privado, en los hogares creando la ilusión de que somos uno más del grupo social, lo que Bion ${ }^{16}$, denomina los supuestos básicos del grupo como un sentimiento de euforia y de unión que se siente en algunos momentos.

Como decíamos, en los primeros días del Estado de Alarma predominaba el sentimiento de "estar todos navegando en la misma dirección" con esa sensación de ser uno con el grupo aplaudiendo cada tarde para apoyar a los sanitarios y otros profesionales que están en primera línea, compartiendo canciones, recetas, bailes, ocurrencias, ánimos... aunque al final siempre quedase una sensación de soledad e impotencia ante una situación que nos desborda y nos hace sentir muy insignificantes. 
Ya decía Bion que no hace falta que los individuos estén reunidos para que los fenómenos grupales existan, todos los individuos forman parte de un grupo aunque se sientan aislados o marginados dentro de él, y por lo tanto sus conductas y reacciones deben estudiarse dentro de los contextos grupales actuales e históricos.

Estamos asistiendo a un hecho importante, la necesidad de sentirse cohesionado con la comunidad, volver a valorar los ritos, no como algo nostálgico, de hecho puede sonar a un acto pasado de moda e incluso no percibirse como algo necesario actualmente, sin embargo, el rito es el que nos trae la percepción simbólica, tal como nos explica Hans, 2020. En nuestra sociedad actual podemos sentir que estamos conectados, que somos auténticos, que nos podemos representar a nosotros mismos pero lo que nos va a ayudar al sentido de comunidad, a hacer habitable el tiempo y el espacio en el que vivimos, en palabras de Hans, 2020, pp 29, es el hecho de que...

... el proyecto de la propia identidad no debería ser egoísta y tendría que hacerse teniendo de fondo un horizonte semántico social que le otorgara una relevancia más allá del propio yo.

Necesitamos de rituales y símbolos que nos ayuden a soportar esta situación que no entendemos y que nos pone a prueba como individuos y por lo tanto como sociedad.

Una viñeta clínica muy significativa es el caso de la paciente María (nombre supuesto) ya en la fase casi final de su terapia. "María sufrió la pérdida de su padre en las primeras semanas del Estado de Alarma, no le pudo acompañar ni despedirse de él porque estaba aislado en una residencia de mayores, tampoco pudo organizar el entierro hasta que no pasaron unos meses, y cuando lo trae a las sesiones el terapeuta también está en duelo por su madre que había fallecido en la misma época, María no lo sabe pero los sentimientos están ahí, el terapeuta no es partidario de revelar su vida privada pero se da cuenta que en este caso puede hacerlo, son muchos años de relación terapéutica, hay un buen vínculo y el hecho de revelar ${ }^{17}$ este duelo personal ayuda a la paciente a sentirse reconocida en un dolor que sentía como algo solitario, que no podía compartir ni iba a entender nadie, pues el ritual tan importante de la despedida y el entierro no había tenido lugar y ella se sentía extraña en medio de una situación extraña, lo que duró hasta que en la primera desescalada pudieron reunir a la familia y organizar el ritual del entierro, y también ayudó el hecho de participar en un periódico que hizo un homenaje a los fallecidos en esa residencia en tiempos de confinamiento total, con fotos y relatos"

Los terapeutas nos estamos enfrentando a nuevos desafíos que exigen poner en práctica cambios en el marco teórico (que ya se están estudiando e investigando desde la época postfreudiana $)^{18}$ : como el encuadre, la revisión de los conceptos psicoanalíticos clásicos del inconsciente y la psicopatología; la implantación progresiva de la teoría del trauma; las fantasías sobre uno mismo y el mundo; la escucha intersubjetiva sin olvidar la dimensión 
intrasubjetiva; la actitud ante la vida así como la necesidad de abordar la culpa y el duelo de todo ser querido que siempre se acompaña de un desgarramiento, una ruptura; y en este caso también el abordaje del "duelo colectivo" que nos hace pensar en los rituales tan necesarios para sentir que nuestro self transciende más allá de nosotros mismos y de la sociedad y el tiempo y espacio que habitamos.

Hoy más que nunca el terapeuta necesita tanto de sus conocimientos, y de su experiencia clínica como de su experiencia personal, sus vivencias y su curiosidad por la condición humana. Como decía Pessoa, 2006, pp $256 \ldots$.

... Nos cansamos de todo, salvo de comprender. A veces no resulta fácil descubrir el sentido de la frase...

Pero en medio de tanta incertidumbre seguimos en un esfuerzo por ofrecer la mejor calidad de asistencia y de comprensión del ser humano.

\section{REFERENCIAS}

Ávila Espada, A (ed.) (2013). La tradición interpersonal. Perspectiva social y cultural en psicoanálisis. Col. Pensamiento Relacional. Vol. 8. Ágora, Madrid.

Bauman, Z. (2010). La globalización. Consecuencias humanas. Fondo de cultura Económica. De la primera edición en ingles de 1998 The Globalization. The Human Conseguences. Traducción de Daniel Zadunaiskya

Bion, W.R. (1974). Experiencias en grupos. Editorial Paidós. Buenos Aires

Castaño, R. (2011). La terapia sexual, una mirada relacional. Col. Pensamiento Relacional. Vol. 4. Ágora, Madrid.

Coderch, J. (2012). Realidad, interacción y cambio psíquico. La práctica de la psicoterapia relacional II. Col. Pensamiento Relacional. Vol. 5. Ágora, Madrid.

Coderch, J. (2014). Avances en Psicoanálisis Relacional. Nuevos campos de exploración para el psicoanálisis. Col. Pensamiento Relacional. vol. 10. Ágora, Madrid.

Freud, S. (1981). Obras completas $4^{\text {a }}$ edición. Biblioteca Nueva. Traducción del alemán de Luis López Ballesteros y de Torres. Tomos I, II y III

Freud, S. (1911). Los dos principios del funcionamiento mental. En Ibid.

Gadamer, H.G. (2017). El estado oculto de la salud. Gedisa editorial. Del título original en alemán Über die Verborgenheit der esundheit de 1993. Traducción Nélida Machain.

Garcés, M. (2013). Un mundo común. Edicions Bellaterra.

Hans, B.(2020). La desaparición de los rituales. Col. Pensamiento Herder, dirigida por Manuel Cruz. Herder editorial. 
Lipovetsky, G. (2008). La sociedad de la decepción. Entrevista con Bertrand Richard. Col. Argumentos. Anagrama.

Mitchell, A. S. (1993). Conceptos relacionales en psicoanálisis. Una integración. Siglo XXI editores, de la primera edición en inglés de 1988. Titulo original Relational concepts in psychoanalysis, an integration.

Mitchell, A.S; Black, M.J. (2004). Más allá de Freud, una historia del pensamiento psicoanalítico moderno. Herder

Orange,D.M; Atwood,G.E; Stolorow,R.D. (2012). Trabajando intersubjetivamente. Contextualismo en la práctica psicoanalítica. Col. Pensamiento Relacional vol. 6 Madrid: Ágora Relacional

Peteiro, J. (2010). El autoritarismo científico. Miguel Gómez ediciones.

Pessoa, F. (2006). Libro del desasosiego. El Acantilado. Traducción de Perfecto. E. Cuadrado.

Rodríguez Sutil, C. (2010). Narcisismo de piel fina y narcisismo de piel dura. Comentarios adicionales sobre el narcisismo. Clínica e Investigación Relacional. sección Debates sobre el narcisismo. Enlace www.ceir.org.es

Rodríguez Sutil, c. (2014). Psicopatología psicoanalítica relacional. La persona en relación y sus problemas. Col. Pensamiento Relacional. Vol. 12. Ágora, Madrid.

Sánchez-López M del P, Dresch V. (2008). The 12-Item General Health Questionnaire (GHQ-12): reliability, external validity and factor structure in the Spanish population. Psicothema 2008 Nov; $20(4): 839-43$

Stolorow, R; Atwood, G. (2004). Los contextos del ser, las bases intersubjetivas de la vida psíquica, Herder Barcelona.

Tizón, J. L. (2013). Pérdida, Pena, Duelo. Vivencias, investigación y asistencia. Selección Salud Mental. Fundació Vidal i Barraquer. Herder editorial.

Original recibido con fecha: 22/1/2021 Revisado: 20/3/2021 Aceptado: 30/03/2021

NOTAS:

${ }^{1}$ La tercera ola del coronavirus se produce después de las fiestas navideñas que supusieron una relajación temporal de las medidas de seguridad.

${ }^{2}$ Según datos del Instituto Nacional de Estadística (INE) el confinamiento ha disparado el uso de Internet en España, que ha aumentado en más de un millón de usuarios, hasta llegar a un total de 32, 8 millones, https://www.ine.es/

${ }^{3}$ La Organización Mundial de la Salud en sus siglas en español OMS: Síndrome de fatiga pandémica COVID19; enlace https://www.who.int/es

4 El Estado de alarma se declara en España en el B.O.E por Real Decreto 463/2020 de 14 de marzo y se prorroga por Real Decreto 476/2020 de 27 de marzo.

5 Han participado en este estudio online todas las secciones de la Federación Española de Asociaciones de Psicoterapeutas en sus siglas FEAP, http://www.feap.es/ con sus diferentes asociaciones, y sus resultados se presentarán en el próximo Congreso Nacional de FEAP que está previsto se organice en Bilbao, en noviembre de 2021. Sección Psicoanalíticas de Niños y Adolescentes; Sección de Familia; Sección de Sistémica; Sección de

@ ( Derechos reservados/Copyright de Clínica e investigación Relacional y los autores. Prohibida la reproducción total o parcial sin autorización expresa. Este material es para uso científico y profesional exclusivamente y puede contener información clínica sensible. Los editores no se responsabilizan de los contenidos de los autores. Dirigir las consultas sobre derechos y autorizaciones a ceir@psicoterapiarelacional.es 
Psicoterapias Humanistas; Sección Psicoterapias Psicoanalíticas; y Sección Cognitivo Conductual. Accesible aquí: http://ow.ly/2yyzzormvYZ

6 Rodríguez Sutil, C. (2014) ver pp 230 y ss. Sobre las emoción, motivación y teorías psicológicas ¿son las emociones racionales o irracionales?.

7 Según el informe COTS del estudio "Condiciones de trabajo, inseguridad y salud de los y las trabajadoras residentes en España en el contexto del COVID-19", realizado por la Universidad de Barcelona a 20.000 personas, durante la primera ola de la pandemia, se incrementó en consumo de psicofármacos hasta el $20 \%$ de la población, siendo el 12\% nuevas prescripciones. En nuestro estudio, el consumo de psicofármacos fue del 11\%. Accesible aquí: https://istas.net/sites/default/files/2021-01/InformeCOTS 01072020-1 1.pdf

${ }^{8}$ Esta pandemia ha traído nuevos términos de uso cotidiano, como: confinamiento, desescalada, primera ola, segunda ola, tercera ola, reconfinamiento, ...

9 Para entender la implantación progresiva de la teoría del trauma en psicoanálisis ver autores como Sullivan; Fromm; Horney; Mitchell; Aron; Benjamin; (Coderch, 2012, pp 302)

${ }^{10}$ En el mes de marzo 2021 se activará una segunda parte del estudio FEAP COVID-19 para realizar una comparación del impacto de la pandemia en los psicoterapeutas, un año después de que se impusiera el Estado de Alarma.

${ }^{11}$ Es interesante destacar las dos formas de considerar la intersubjetividad: 1) los autores de la teoría de la intersubjetividad, Orange, Atwood, Stolorow, Lachmann... que defienden la intersubjetividad como el campo en el que intervienen dos subjetividades; y 2) la escuela relacional, Mitchell, Aron, Benjamin, Stern... que defienden la intersubjetividad como un proceso dinámico que va desde la negación del otro al reconocimiento del otro como un self igual pero diferente (Ver Coderch, 2014)

${ }^{12} \mathrm{GHO}_{12}$, es un instrumento, validado en España, que ayuda a detectar el impacto negativo en la salud mental

${ }^{13}$ Un $5 \%$ es el porcentaje de la población general que se había infectado de la COVID-19 en España en estas fechas. Accesible aquí: https://portalcne.isciii.es/enecovid19/informes/informe_final.pdf

${ }^{14}$ De acuerdo con Tizón, 2013, pp 410 ... como seres humanos todos tenemos el deber de ayudar en los duelos de los demás, y el derecho a que nos ayuden en los nuestros ... la principal ayuda para el duelo es la que puede proporcionar los núcleos vivenciales naturales de las personas en duelo y su red social. Este autor distingue entre duelo normal, complicado y patológico lo que compete a la forma de intervención de los profesionales.

${ }^{15}$ El fotoperiodista español Santi Palacios se alzó con el 24 Premio Internacional de Fotografía Humanitaria Luis Valtueña por el trabajo Soledades mayores, por un recorrido visual por las residencias de ancianos de Cataluña durante el Estado de alarma a causa de la pandemia. Los centros de mayores fueron uno de los puntos negros de la emergencia sanitaria en España, uno de los países más afectados por la COVID-19. https://www.medicosdelmundo.org/

${ }^{16}$ En los supuestos básicos del grupo Bion, 1974, hace referencia a los deseos, impulsos, y voluntades unánimes de grupo en un momento dado, y describe 3 supuestos básicos: de dependencia, de apareamiento y de ataque y fuga.

${ }^{17}$ Revelación y/o auto-revelación, dos conceptos que se están debatiendo en Psicoanálisis Relacional en relación con lo que se ha denominado el enactment y que tienen que ver con la revisión de los conceptos clásicos de transferencia - contratransferencia, con la influencia del pasado en el presente tanto en paciente como terapeuta, y con la interacción paciente - terapeuta como fundamento del cambio psíquico (Ver Coderch, 2012, pp 137165)

18 La etapa postfreudiana se inicia ya desde los primeros años treinta del pasado siglo, aunque desafortunadamente la pluralidad, la tradición interpersonal y sociocultural en Psicoanálisis no ha sido apreciada más allá de la costa Este de los Estados Unidos... (ver Ávila, 2013)

@ Derechos reservados/Copyright de Clínica e investigación Relacional y los autores. Prohibida la reproducción total o parcial sin autorización expresa. Este material es para uso científico y profesional exclusivamente y puede contener información clínica sensible. Los editores no se responsabilizan de los contenidos de los autores. Dirigir las consultas sobre derechos y autorizaciones a ceir@psicoterapiarelacional.es 